\title{
Yield criterion for a perforated sheet with a uniform triangular pattern of round holes and a low ligament ratio
}

\author{
Yi-Che Lee, Fuh-Kuo Chen ${ }^{*}$ \\ Department of Mechanical Engineering, National Taiwan University, 1 Roosevelt Road, Sect. 4, Taipei 10764, Taiwan, ROC
}

Received 28 February 1999

\begin{abstract}
The plastic behavior of a perforated sheet with round holes in a triangular pattern depends largely on the hole size of the perforated sheet. This study investigates the plastic behavior of perforated sheets with low ligament ratios. A yield criterion for the perforated sheets is also proposed in terms of apparent stresses by employing the equivalent-continuum approach. The effectiveness of the proposed yield criterion is then demonstrated by comparing the predicted values of apparent yield stresses and apparent strain ratios with results obtained from finite-element analysis and experiment. (C) 2000 Elsevier Science S.A. All rights reserved.
\end{abstract}

Keywords: Perforated sheet; Equivalent continuum; Finite element method

\section{Introduction}

Tube sheets in heat-exchanger equipment [1] and shadow masks in high-resolution color picture tubes [2] are made of perforated sheets. Properly designing and manufacturing of tube sheets and shadow masks depends on characterizing the plastic behavior of the perforated sheets. More specifically, defining a yield criterion and establishing the flow rule are essential in investigating the plastic behavior of perforated sheets.

Chen and coworkers [3,4] and Baik et al. [5-7] analyzed the deformation behavior of perforated sheets. Their analytical work assumed that not only the yielding of a perforated sheet occurs in the base metal at the minimum ligament area, but also that the von Mises yield criterion governs the yielding of the base metal of the perforated sheet. The yield criteria proposed by Chen [4] and Baik et al. [7] are considered applicable over the whole range of ligament ratio. According to Fig. 1, the ligament ratio $\rho$ is defined as the ratio of the ligament width $W$ to the perforated pitch $P$ in a unit cell ABCD. However, the yield criterion as defined by Chen is inappropriate for a perforated sheet with a low ligament ratio. On the other hand, the apparent strain ratios

\footnotetext{
* Corresponding author. Tel.: +886-2-2366-1322; fax: +886-2-2363-1755.

E-mail address: fkchen@w3.me.ntu.edu.tw (F.-K. Chen)
}

predicted by the related flow rule defined by Baik et al. do not correlate well with those obtained in experiments under uniaxial loading for a perforated sheet with a low ligament ratio.

The yielding of a perforated sheet depends on the ligament ratio of the perforated sheet and the loading states. Hence, the plastic behavior of a perforated sheet is extremely complicated, thereby making it difficult to propose a yield criterion for a perforated sheet over the whole range of ligament ratios. In general uses of perforated sheets, e.g. tube sheets in heat-exchanger equipment and shadow-masks in high-resolution color picture tubes, the ligament ratios of the perforated sheets are in the low range. Hence, this study investigates the plastic behavior of perforated sheets with a uniform triangular pattern and low ligament ratios, a yield criterion also being proposed for the perforated sheets.

The plastic behavior of a perforated sheet cannot be characterized by treating each hole because a perforated sheet has a large number of holes. The plastic behavior of a perforated sheet is analyzed by treating the perforated sheet metal as an equivalent continuum that is compressible and anisotropic [8,9]. A yield criterion and associated flow rules are defined in terms of the apparent stresses and the apparent strains to account for the plastic behavior of the perforated sheet. The apparent stress is defined as the external force divided by the equivalent area upon which the force is applied and the apparent strain as the elongation per unit 


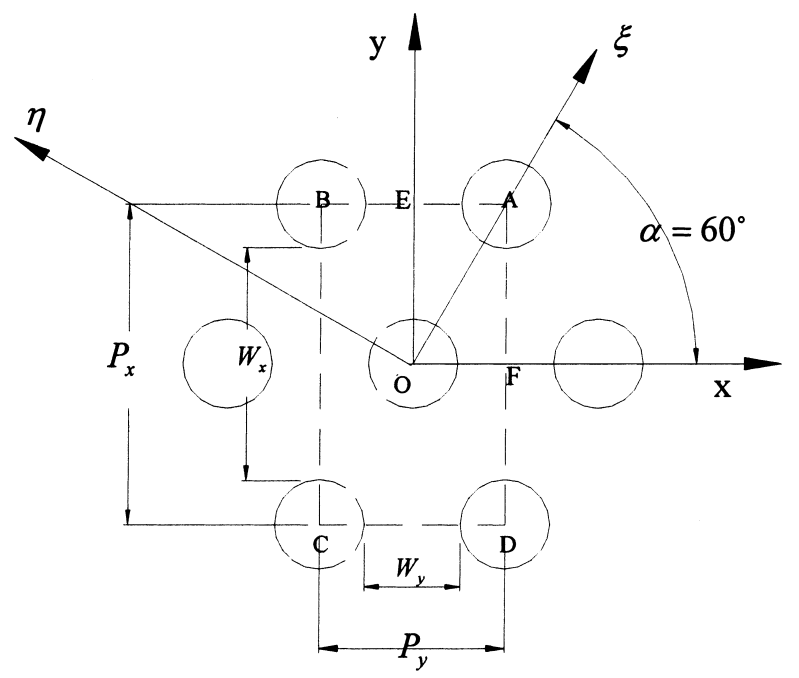

Fig. 1. Round holes arranged in a triangular pattern.

equivalent length. Next, the apparent yield stresses and the apparent strain ratios obtained by the finite-element method and from experiments are used to examine those predicated by the proposed yield criterion and the related flow rule.

\section{Earlier yield criteria for perforated sheets}

Chen [4] and Baik et al. [7] proposed yield criteria for perforated sheets with round holes in a triangular pattern, the concept of an equivalent-continuum was used for analyzing the plastic behavior. In addition, the yielding of a perforated sheet was assumed to be in the minimum ligaments along $\mathrm{AB}$ and $\mathrm{AO}$, as illustrated in Fig. 1. Then, yield criteria for perforated sheets were defined by considering the yield stress states of the minimum ligaments along $\mathrm{AB}$ and $\mathrm{AO}$.

Chen also considered the apparent volume change of an equivalent continuum during the deformation process owing to the volume change of the holes of a perforated sheet. Hence, in addition to the hydrostatic pressure producing plastic deformation, the effect of hydrostatic pressure is also modeled in the general yield criterion for anisotropic materials proposed by Hill [10]. In the plane-stress condition, the form of the yield criterion can be simplified to [4]:

$F S_{x}^{2}+H S_{x} S_{y}+G S_{y}^{2}+N S_{x y}^{2}=Y_{\mathrm{b}}^{2}$,

where $S_{x}$ and $S_{y}$ are the apparent normal stresses in $x$ and $y$ directions, respectively, $S_{x y}$ an apparent shear stress, $Y_{\mathrm{b}}$ the yield stress of the base metal in the perforated sheet, and $F$, $H, G$ and $N$ are parameters. Chen developed two deformation models, i.e. the yielding of a perforated sheet in the minimum ligaments along $\mathrm{AB}$ and $\mathrm{AO}$, to determine the coefficients in the yield criterion equation (1).

In the two deformation models, the stresses are assumed to be uniform across the ligament widths. In one model, with respect to yielding in the minimum ligament along $\mathrm{AB}$, the mean true stresses $\sigma_{x}$ and $\sigma_{y}$ in the base metal of the minimum ligament along $\mathrm{AB}$ are expressed by

$\sigma_{x}=\frac{S_{x}}{\rho_{x}}, \quad \sigma_{y}=\frac{S_{y}}{\rho_{y}}$,

where $\rho_{x}=W_{x} / P_{x}$ and $\rho_{y}=W_{y} / P_{y}$. In another model, with respect to yielding in the minimum ligament along $\mathrm{AO}$, the mean true stresses $\sigma_{\xi}$ and $\sigma_{\eta}$ in the base metal of the minimum ligament along $\mathrm{AO}$ are obtained by transforming the apparent stresses $S_{x}$ and $S_{y}$ into stresses in $\xi-\eta$ coordinates, as illustrated in Fig. 1:

$\sigma_{\xi}=\frac{1}{4 \rho_{\xi}}\left(S_{x}+3 S_{y}\right), \quad \sigma_{\eta}=\frac{1}{4 \rho_{\eta}}\left(3 S_{x}+S_{y}\right)$,

$\sigma_{\xi \eta}=-\frac{\sqrt{3}}{4 \rho_{m}}\left(S_{x}-S_{y}\right)$,

where $\sigma_{\xi}=\rho_{x}, \sigma_{\eta}=\rho_{y}$ and $\rho_{m}=\frac{1}{2}\left(\rho_{x}+\rho_{y}\right)$. By substituting Eqs. (2) and (3) into the von Mises yield criterion, the following yield equations are derived.

For yielding of the minimum ligament along $\mathrm{AB}$ :

$\left(\frac{1}{\rho_{x}^{2}}\right) S_{x}^{2}-\left(\frac{1}{\rho_{x} \rho_{y}}\right) S_{x} S_{y}+\left(\frac{1}{\rho_{y}^{2}}\right) S_{y}^{2}=Y_{\mathrm{b}}^{2}$.

For yielding of the minimum ligament along AO:

$$
\begin{aligned}
\frac{1}{16}\left(\frac{1}{\rho_{x}^{2}}-\frac{3}{\rho_{x} \rho_{y}}+\frac{9}{\rho_{y}^{2}}+\frac{9}{\rho_{m}^{2}}\right) S_{x}^{2} \\
\quad-\frac{1}{16}\left(-\frac{6}{\rho_{x}^{2}}+\frac{10}{\rho_{x} \rho_{y}}-\frac{6}{\rho_{y}^{2}}+\frac{18}{\rho_{m}^{2}}\right) S_{x} S_{y} \\
\quad+\frac{1}{16}\left(\frac{9}{\rho_{x}^{2}}-\frac{3}{\rho_{x} \rho_{y}}+\frac{1}{\rho_{y}^{2}}+\frac{9}{\rho_{m}^{2}}\right) S_{y}^{2}=Y_{\mathrm{b}}^{2}
\end{aligned}
$$

Chen's [4] analytical work assumed that the normal stresses are uniform across the ligament width. However, the normal stresses in the $x$ - and $\xi$-directions are not close to being uniform, particularly for a perforated sheet with a low ligament ratio, owing to the various boundary conditions across the ligament width, as indicated in Fig. 2.
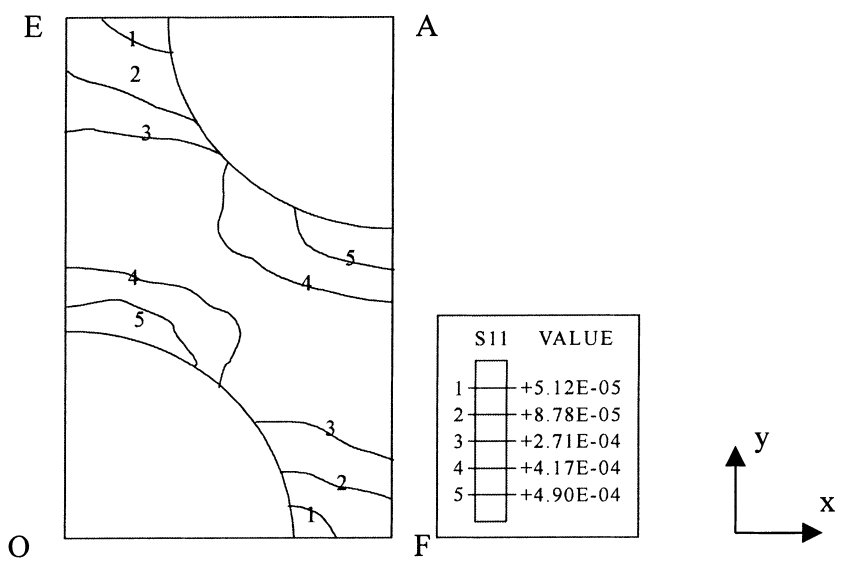

Fig. 2. The distribution of normal stress $\sigma_{x}$ under biaxial loading. 
On the other hand, Baik et al. [7] defined the yield stress of a perforated sheet as the apparent flow stress at which the center of a ligament begins to yield. Then, the normal stresses, $\sigma_{x}$ and $\sigma_{\xi}$, across the ligament width are corrected and can be calculated by elastic theory [11] as

$\sigma_{x}=\frac{f}{\rho_{x}} S_{x}, \quad \sigma_{\xi}=\frac{f}{4 \rho_{x}}\left(S_{x}+3 S_{y}\right)$,

where factor $f$ is a function of the ligament ratio. Substituting the corrected stresses into the von Mises yield criterion allows one to obtain the following yield criteria.

For yielding along $\mathrm{AB}$ :

$\left(\frac{f}{\rho_{x}}\right)^{2} S_{x}^{2}-\left(\frac{f}{\rho_{x} \rho_{y}}\right) S_{x} S_{y}+\left(\frac{1}{\rho_{y}^{2}}\right) S_{y}^{2}=Y_{\mathrm{b}}^{2}$.

For yielding along AO:

$$
\begin{aligned}
& \frac{1}{16}\left[\left(\frac{f}{\rho_{x}}\right)^{2}-\frac{3 f}{\rho_{x} \rho_{y}}+\frac{9}{\rho_{y}^{2}}+\frac{9}{\rho_{y}^{2}}\right] S_{x}^{2} \\
& +\frac{1}{16}\left[6\left(\frac{f}{\rho_{x}}\right)^{2}-\frac{10 f}{\rho_{x} \rho_{y}}+\frac{6}{\rho_{y}^{2}}-\frac{18}{\rho_{y}^{2}}\right] S_{x} S_{y} \\
& +\frac{1}{16}\left[9\left(\frac{f}{\rho_{x}}\right)^{2}-\frac{3 f}{\rho_{x} \rho_{y}}+\frac{1}{\rho_{y}^{2}}+\frac{9}{\rho_{y}^{2}}\right] S_{y}^{2}=Y_{\mathrm{b}}^{2} .
\end{aligned}
$$

The parameter $f$ is approximately zero for low ligament ratios but increases to unity with increasing ligament ratio. Hence, the normal stresses $\sigma_{x}$ and $\sigma_{\xi}$ in Eq. (6) are close to zero. The stresses are substituted in the yield criterion equations (7) to obtain the coefficient of the $S_{x} S_{y}$ term, being about zero. As a result, the apparent strain ratio under uniaxial loading, $S_{x}=0$, predicated by the yield criterion [7] is very small or negative. The outcome is unreasonable during deformation under uniaxial loading. Therefore, the yield criteria proposed by Chen and Baik et al. are inappropriate for perforated sheets with low ligament ratios. In this study, we propose a yield criterion to accurately describe the plastic behavior of perforated sheets with low ligament ratios.

\section{A yield criterion for a perforated sheet}

This study investigates perforated sheets with a uniform triangular pattern of round holes and of low ligament ratios. The yielding of the perforated sheets is also assumed to occur in the minimum ligament along AO under uniaxial and biaxial loadings. Next, the average values of the stress components across the ligament width along AO are calculated to define the yield stress states of a perforated sheet. However, the definition of stress states differs from those in Chen [4] and Baik et al. [7].

\subsection{Yield criteria for perforated sheets}

For stress analysis of the minimum ligament along $\mathrm{AO}$, as shown in Fig. 1, the normal stress $\sigma_{\eta}$ and the shear stress $\sigma_{\xi \eta}$ across the ligament width along $\mathrm{AO}$ are assumed to be uniform because the ligament width is narrow for low ligament ratios. Under biaxial loading in the $x$ - and $y$ directions, according to Fig. 1, the apparent stresses $S_{x}$ and $S_{y}$ are transformed into stresses in $\xi-\eta$ coordinates. The apparent stresses in $\xi-\eta$ coordinates can be obtained as $S_{\xi}=\frac{1}{4}\left(S_{x}+3 S_{y}\right), \quad S_{\eta}=\frac{1}{4}\left(3 S_{x}+S_{y}\right)$, $S_{\xi \eta}=-\frac{\sqrt{3}}{4}\left(S_{x}-S_{y}\right)$.

Moreover, the stresses $\sigma_{\eta}$ and $\sigma_{\xi \eta}$ across the minimum ligament width along $\mathrm{AO}$ can be defined as

$\sigma_{\eta}=\frac{S_{\eta}}{\rho_{y}}=\frac{1}{4 \rho_{y}}\left(3 S_{x}+S_{y}\right)$,

$\sigma_{\xi \eta}=\frac{S_{\xi \eta}}{\rho_{y}}=-\frac{\sqrt{3}}{4 \rho_{y}}\left(S_{x}-S_{y}\right)$.

On the other hand, the distribution of transverse normal stress $\sigma_{\xi}$ varies across the ligament width along AO and can be derived by use of the theoretical analysis of Isida [12]. Whilst analyzing the stress distribution of a uniform triangular pattern of round holes in an infinite solid [13], appropriate unit regions are initially chosen and then the complex stress potentials, $\varphi(z)$ and $\phi(z)$, are assumed in the form of a Laurent series expansion satisfying the traction-free conditions along the hole edges. Stress components are expressed in terms of $\varphi(z), \phi(z)$ and their derivatives as the following:

$\sigma_{\xi}+\sigma_{\eta}=4 \operatorname{Re}\left[\phi^{\prime}(z)\right]$

$\sigma_{\xi}-\sigma_{\eta}+2 \mathrm{i} \sigma_{\xi \eta}=2\left[\bar{z} \phi^{\prime \prime}(z)+\varphi^{\prime}(z)\right]$.

The complex stress potentials in the form of a Laurent series expansion are expressed by

$\phi(z)=\sum_{n=0}^{\infty}\left(A_{2 n} z^{2 n+1}+B_{2 n} z^{-2 n-1}\right)$,
$\varphi(z)=\sum_{n=0}^{\infty}\left(C_{2 n} z^{2 n+1}+D_{2 n} z^{-2 n-1}\right)$,

where $\varphi(z)$ and $\phi(z)$ are two complex stress potentials that must be analytical in the triangular unit region $(\triangle \mathrm{OMN})$ chosen, as shown in Fig. 3, and $z=\xi+\mathrm{i} \eta$. Next, the unknown coefficients $A_{2 n}, B_{2 n}, C_{2 n}$ and $D_{2 n}$ in the Laurent series are determined by considering the traction-free condition along the hole edge and the boundary conditions at the outer edges of the triangular unit regions used [13]. Numerical calculations are carried out based on element-wise resultant forces and displacements [12]. The stress distributions in the base material of the triangular region can be obtained under loading in the $\xi$ direction. In addition, the average value of the transverse normal stress $\sigma_{\xi}$ across the ligament width along $\mathrm{OM}$ is expressed by

$\sigma_{\xi}=\frac{1}{\left|M_{0} M\right|} \int_{M_{0}}^{M}\left[\sigma_{\xi}\right]_{\eta=0} \mathrm{~d} \xi=\frac{k}{\rho_{y}} S_{\xi}=\left(\frac{k}{\rho_{y}}\right)\left(\frac{1}{4} S_{x}+\frac{3}{4} S_{y}\right)$, 

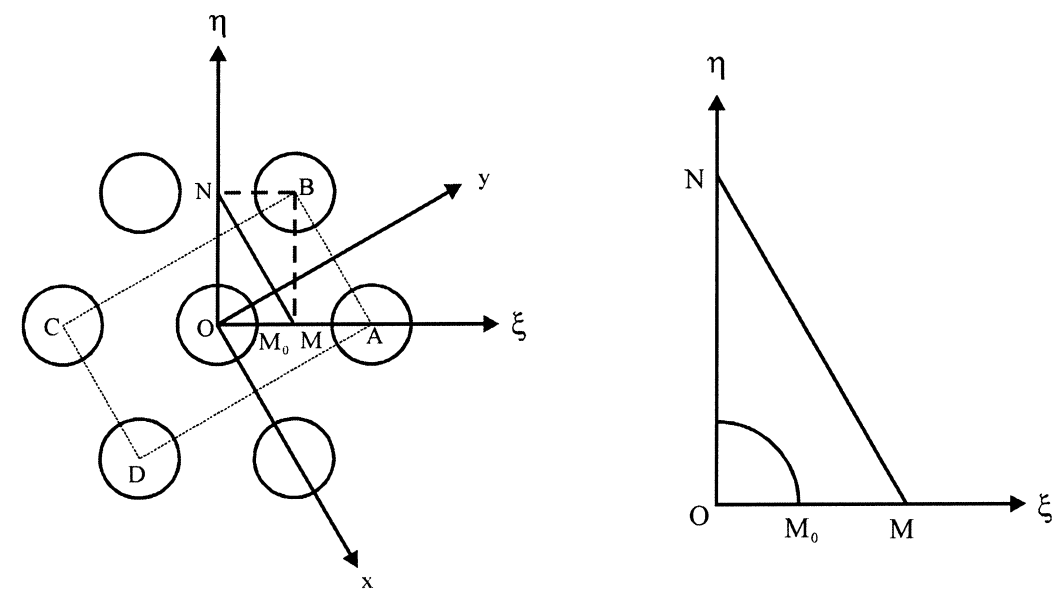

Fig. 3. Triangular unit region $(\triangle \mathrm{OMN})$.

where $\left[\sigma_{\xi}\right]_{\eta=0}$ denotes the stress distribution in the $\xi$ direction along $\mathrm{M}_{0} \mathrm{M}$ and $k$ is a function of the ligament ratio that can be calculated following the method proposed in [13], the result being shown in Fig. 4.

The von Mises yield criterion under the plane-stress condition applies for the base metal of the perforated sheet:

$\sigma_{\eta}^{2}-\sigma_{\xi} \sigma_{\eta}+\sigma_{\xi}^{2}+3 \sigma_{\eta \xi}^{2}=Y_{\mathrm{b}}^{2}$.

The stresses in Eqs. (10), (11) and (16) are then substituted in the von Mises yield criterion equation (17), whereby the yield criterion for the perforated sheets can be obtained as

$$
\begin{aligned}
& \left(\frac{1}{4 \rho_{y}}\right)^{2}\left[\left(k^{2}-3 k+18\right) S_{x}^{2}+\left(6 k^{2}-10 k-12\right) S_{x} S_{y}\right. \\
& \left.\quad+\left(9 k^{2}-3 k+10\right) S_{y}^{2}\right]=Y_{\mathrm{b}}^{2} .
\end{aligned}
$$

Comparing Eqs. (1) and (18) allows the determination of coefficients $F, G$ and $H$ as

$$
\begin{aligned}
& F=\left(\frac{1}{4 \rho_{y}}\right)^{2}\left(k^{2}-3 k+18\right), \\
& G=\left(\frac{1}{4 \rho_{y}}\right)^{2}\left(6 k^{2}-10 k-12\right), \\
& H=\left(\frac{1}{4 \rho_{y}}\right)^{2}\left(9 k^{2}-3 k-10\right) .
\end{aligned}
$$

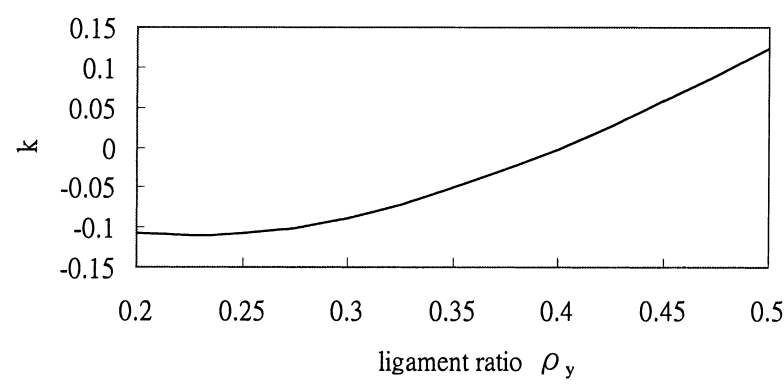

Fig. 4. The parameter $k$ as a function of ligament ratio $\left(\rho_{y}\right)$.
If the hole size is zero, $\rho_{y}=\rho_{x}=1$, the apparent stresses of a perforated sheet are the same as the mean true stresses in the base metal of the sheet and $k$ in Eq. (16) is equal to unity. By allowing the $x$ - and $y$-axes to be the principal axes, the yield criterion is reduced to the von Mises yield criterion, $F=1$, $G=-1$, and $H=1$. This observation suggests that the yield criterion function corresponds to the limiting case for a solid isotropic material.

\subsection{Yield locus}

Fig. 5 plots the yield locus according to the yield criterion proposed above on the stress plane for a ligament ratio, $\rho_{y}$ of 0.213 . The yield locus is smooth and no singular points exist on the yield surface. However, the yield loci plotted by Chen [4] and Baik et al. [7] correspond to two deformation models. Fig. 6 presents both yield loci, which consist of two yield criteria results of singular points on the yield surface.

\subsection{Flow rule and yield stress}

The related flow rules are established by assuming the yield function $f\left(S_{i j}\right)$ to be a plastic potential:

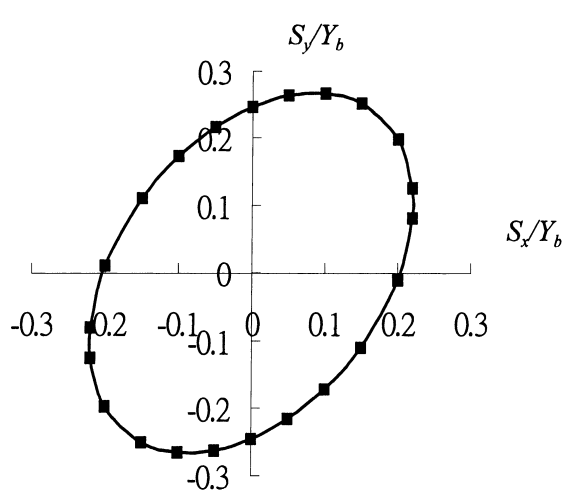

Fig. 5. The yield locus for a ligament ratio $\left(\rho_{y}\right)$ of 0.213 . 


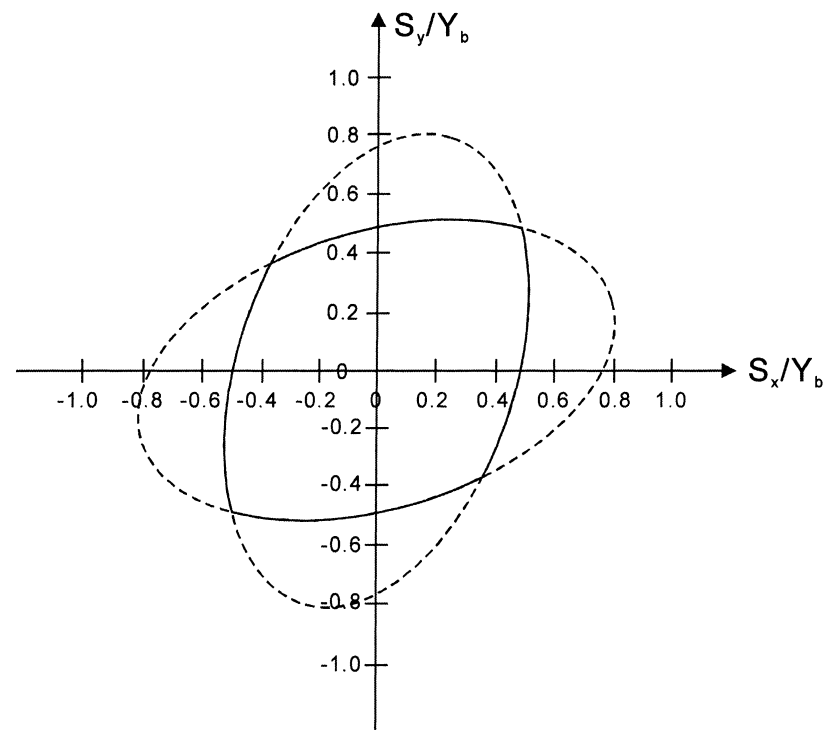

Fig. 6. The yield locus plotted by the two yield criteria given in Ref. [4].

$\dot{\varepsilon}_{i j}=\dot{\lambda} \frac{\partial f}{\partial S_{i j}}$

where $\dot{\varepsilon}_{i j}$ are the apparent strain rates and $\dot{\lambda}$ is a proportionality constant. When the strains remain small, the strain $e_{i j}$ may be substituted for the strain rates [14]. By considering this condition, Eq. (20) becomes

$e_{i j}=\lambda \frac{\partial f}{\partial S_{i j}}$.

The yield function (Eq. (18)), is substituted into the flow rule (Eq. (21)), to obtain

$e_{x}=\lambda\left(\frac{1}{4 \rho_{y}}\right)^{2}\left[2\left(k^{2}-3 k+18\right) S_{x}+\left(6 k^{2}-10 k-12\right) S_{y}\right]$,

$e_{y}=\lambda\left(\frac{1}{4 \rho_{y}}\right)^{2}\left[\left(6 k^{2}-10 k-12\right) S_{x}+2\left(9 k^{2}-3 k+10\right) S_{y}\right]$.

In uniaxial tension in the $y$ direction, $S_{x}=0$, the apparent yield stress $S_{y}$ and the apparent strain ratio $R_{y}$ defined as the ratio of the apparent strain $e_{x}$ in the $x$ direction to the apparent strain $e_{y}$ in the $y$ direction, can be expressed as

$S_{y}=\left(\frac{1}{4 \rho_{y}}\right)\left(9 k^{2}-3 k+10\right)^{-1 / 2} Y_{\mathrm{b}}$,

$R_{y}=-\frac{e_{x}}{e_{y}}=-\frac{\left(6 k^{2}-10 k-12\right)}{2\left(9 k^{2}-3 k+10\right)}$.

Similarly, in uniaxial tension in the $x$ direction, $S_{y}=0$, the apparent yield stress $S_{x}$ and the ratio of the apparent strains
$R_{x}$ are determined by

$$
\begin{aligned}
& S_{x}=\left(\frac{1}{4 \rho_{y}}\right)\left(k^{2}-3 k+18\right)^{-1 / 2} Y_{\mathrm{b}}, \\
& R_{x}=-\frac{e_{y}}{e_{x}}=-\frac{\left(6 k^{2}-10 k-12\right)}{2\left(k^{2}-3 k+18\right)} .
\end{aligned}
$$

This study confirms the effectiveness of the yield criterion and its related flow rule developed herein by finite-element analysis and by uniaxial tension tests in the $x$ - and $y$ directions.

\section{Finite-element analysis}

Theoretical analysis for the global deformation of a perforated sheet must correspond to the behavior of local deformation. To examine the analytical results, the finite-element method is used to analyze the local deformation. Simulation results obtained by the finite-element method are then compared with those predicted by the theoretical analysis and as determined by tensile tests, respectively.

By considering the symmetry in the geometric configuration of a perforated sheet, a repetitive portion of the unit cell, OFAE, as depicted in Fig. 1, is adopted as the analytical pattern. To determine the boundary conditions of the unit cell, the model of a perforated sheet with many holes is analyzed by the finite-element method under uniaxial and biaxial tensile loading. According to Fig. 7, the displacements are uniform at the symmetric lines of the model during the deformation process. Whilst neglecting the free boundary conditions of the outer edges, the boundary conditions of the unit cell are determined by the results of the finite-element analysis, as summarized in Table 1.

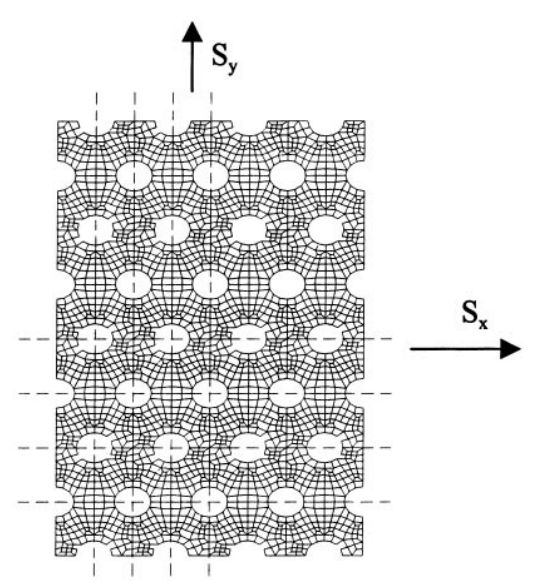

Fig. 7. Uniform displacements at symmetry lines (dash-lines) under biaxial loading (the dash-lines are symmetry lines of the perforated sheet). 
Table 1

The boundary conditions of the unit cell specified in the FEM analysis ${ }^{\mathrm{a}}$

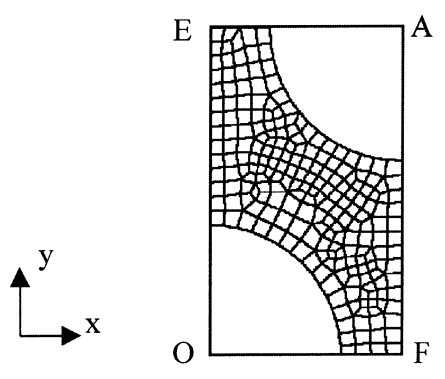

\begin{tabular}{llll}
\hline & EG boundary & AF boundary & AE boundary \\
\hline$x$-axial tension & $U_{x}=0$ & $U_{x}=$ Specified & $U_{y}=$ Uniform \\
$y$-axial tension & $U_{x}=0$ & $U_{x}=$ Uniform & $U_{y}=$ Specified \\
Biaxial tension $(x$ and $y$ axes $)$ & $U_{x}=0$ & $U_{x}=$ Specified & $U_{y}=0$ \\
\hline
\end{tabular}

${ }^{\mathrm{a}} U_{x}$ and $U_{y}$ denote the displacements in the $x$ and $y$ directions, respectively.

In this study, the general purpose finite-element computer code ABAQUS is employed by using a 10-node quadrilateral element to generate the two-dimensional solutions. The steel alloy used in the experiment described in the following section is taken as the simulated material. Young's modulus and Possion's ratio of the material are $198 \mathrm{GPa}$ and 0.3 , respectively. The effective-stress $(\bar{\sigma})$-effective-strain $(\bar{\varepsilon})$ relationship is expressed approximately by $\bar{\sigma}=308$ $+1449 \bar{\varepsilon}(\mathrm{MPa})$.

\section{Experiments}

Experimental results of uniaxial tensile tests, the apparent yield stress and the apparent strain ratio, are used not only to verify the predicted values derived from the theoretical analysis developed herein, but also to compare the results with the values determined by the finite-element method. Herein, the ligament ratios of perforated sheets are in the low range. Hence, the different lower ligament ratios of perforated sheets are prepared for the experimental tests, including the ligament ratios of $0.25,0.3,0.4$ and 0.5 . The test specimens are cut from the $x$ - and $y$-directions in a perforated sheet, as illustrated in Fig. 1. To neglect the effect of the free boundary along the gauge edges of test specimens, at least 10 holes are present in the gauge width. The distance between the centers of two adjacent holes is then recorded to determine the material flow during deformation. The tensile tests are performed with an MTS 810 material test machine, the stretching rate being approximately $1.5 \times 10^{-3} \mathrm{~s}^{-1}$.

After testing, the averaged distance between the centers of two adjacent holes is measured in the loading and transverse direction, and then it is compared with those before testing to obtain the apparent strain ratios. The results of the tensile experiments enable the verification of the apparent strain ratio and the apparent yield stress ratio.

\section{Results and discussion}

Figs. 8 and 9 display the true effective stress contours obtained by the finite-element analysis under uniaxial and biaxial tension for two different ligament ratios, $\rho_{y}=0.285$ and $\rho_{y}=0.515$, respectively. According to Fig. 8, the maximum effective stress is almost in the minimum ligament along AO for a perforated sheet with a low ligament ratio under uniaxial and biaxial tensions. Fig. 9(b) reveals that, when the ligament ratio is greater $\left(\rho_{y}=0.515\right)$ under $y$ direction loading, the maximum effective stress may occur in the minimum ligament along $\mathrm{AB}$ and $\mathrm{AO}$. Hence, the effective stress contours confirm the assumption that the yielding location of a perforated sheet is in the minimum ligament along AO when the ligament ratio $\rho_{y}$ is approximately lower than 0.5 under uniaxial and biaxial loading.

This study proposes a yield criterion to describe the plastic behavior of a perforated sheet with a low ligament ratio. The effectiveness of the proposed yield criterion and related flow rules is demonstrated by comparing the predicated values of the apparent yield stress ratios $\left(Y_{x} / Y_{y}\right)$ and the apparent strain ratios $\left(R_{x}\right.$ and $\left.R_{y}\right)$ with the results obtained from the finite-element analysis and from experimental tests. Fig. 10 summarizes the apparent yield stress ratios predicted by the proposed yield criterion, the finiteelement analysis and the experimental tests. These results are also compared with those predicted by Chen [4] and Baik et al. [7].

Chen's analytical work assumed that the stress distribution is uniform across the minimum ligament width, resulting in an insignificant effect of stress concentration in the minimum ligament [4]. Hence, the predicted stress state that causes yielding at the minimum ligament is too conservative to obtain an accurate value of the apparent yield stresses, particularly under uniaxial loading in the $x$ direction. Chen also indicated that the apparent yield stress in the $x$ direction 
E

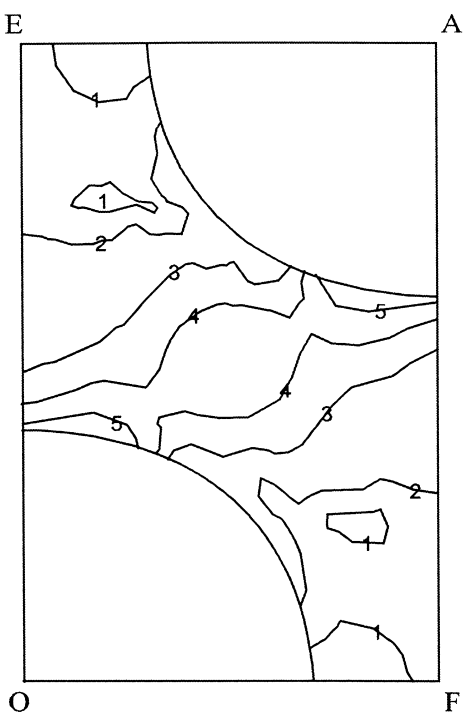

E
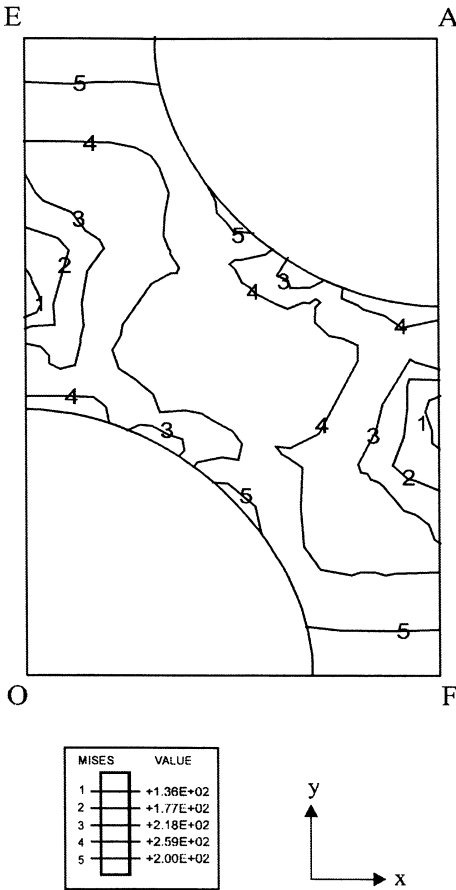

(b)
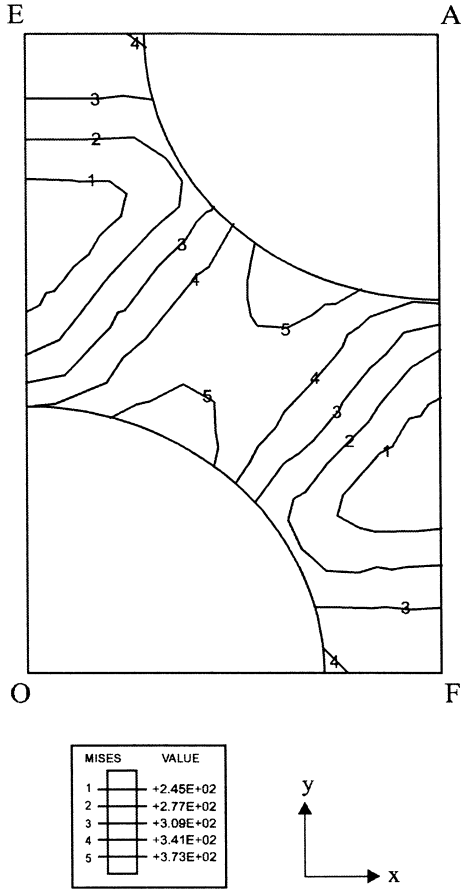

(c)

Fig. 8. The effective stress contours $\left(\rho_{y}=0.285\right)$ : (a) $x$-direction loading; (b) $y$-direction loading; (c) biaxial loading.
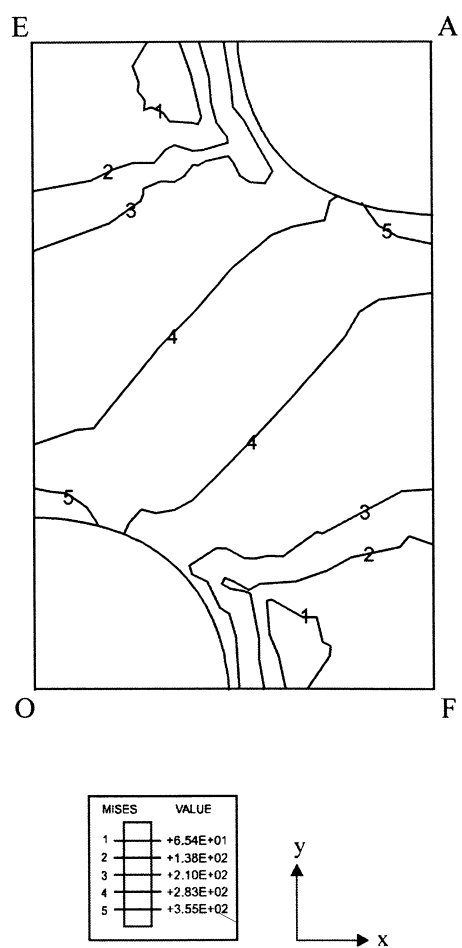

(a)

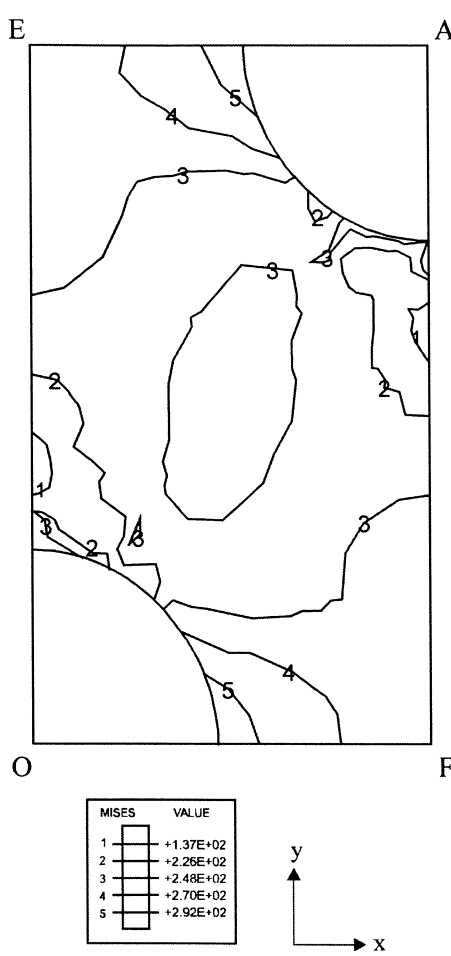

(b)
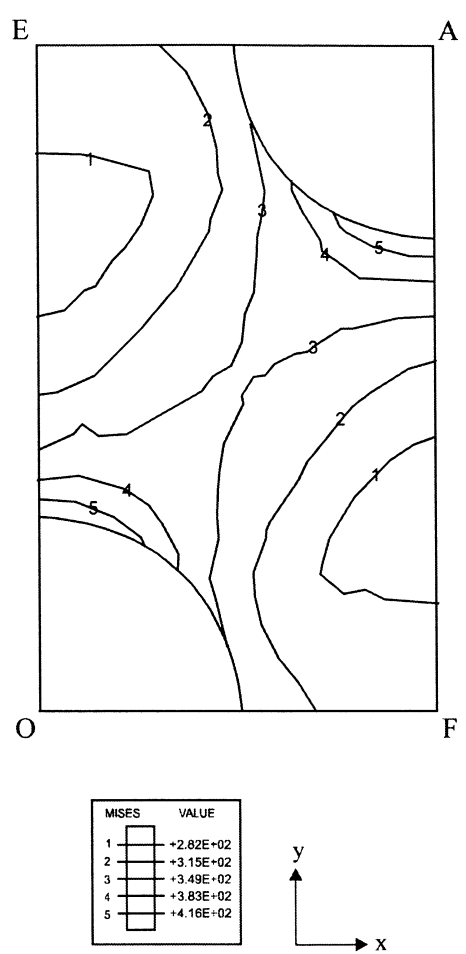

(c)

Fig. 9. The effective stress contours $\left(\rho_{y}=0.515\right)$ : (a) $x$-direction loading; (b) $y$-direction loading; (c) biaxial loading. 


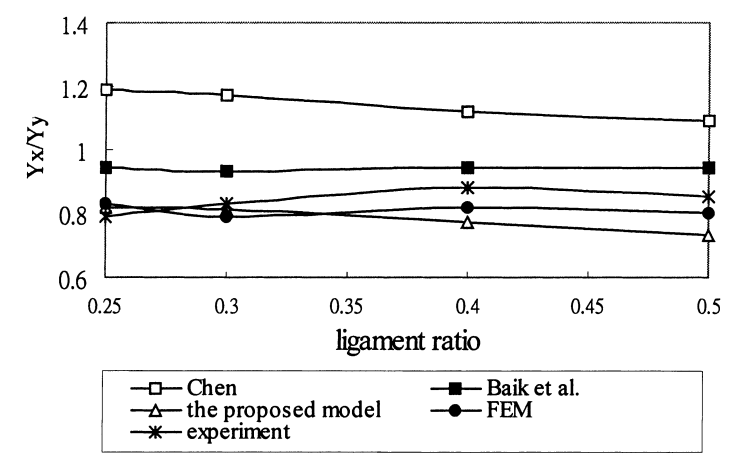

Fig. 10. Comparison of the apparent yield stress ratio $\left(Y_{x} / Y_{y}\right)$.

is greater than the apparent yield stress in the $y$ direction, which is inconsistent with the results obtained by finiteelement analysis and experiment.

On the other hand, the apparent yield stress ratios predicted by Baik et al. and by the present study correlate well with those obtained by finite-element analysis and experimental tests, for greater ligament ratios. However, the predicted results of Baik et al., as shown in Fig. 10, are inappropriate for a perforated sheet with ligament ratios lower than 0.4, compared to those predicted by the present analysis, the finite-element analysis and the experimental tests. Notably, according to Fig. 10, the apparent yield stress ratios predicted by this study are well within the range for a low ligament ratio.

Figs. 11 and 12 present the apparent strain ratios, $R_{x}$ and $R_{y}$, obtained by the theoretical analysis, the finite-element analysis and the experimental tests, respectively. These figures also summarize the results obtained by Chen [4] and Baik et al. [7]. According to Fig. 11, the results predicted by Baik et al. and this study correlate with those obtained by the finite-element analysis and the experimental tests, both in trend and in magnitude. However, under uniaxial loading in the $x$ direction, the uniform stress distribution of the minimum ligament analyzed by Chen is inconsistent with that computed by the finite-element analysis developed herein, particularly for a perforated sheet with a lower

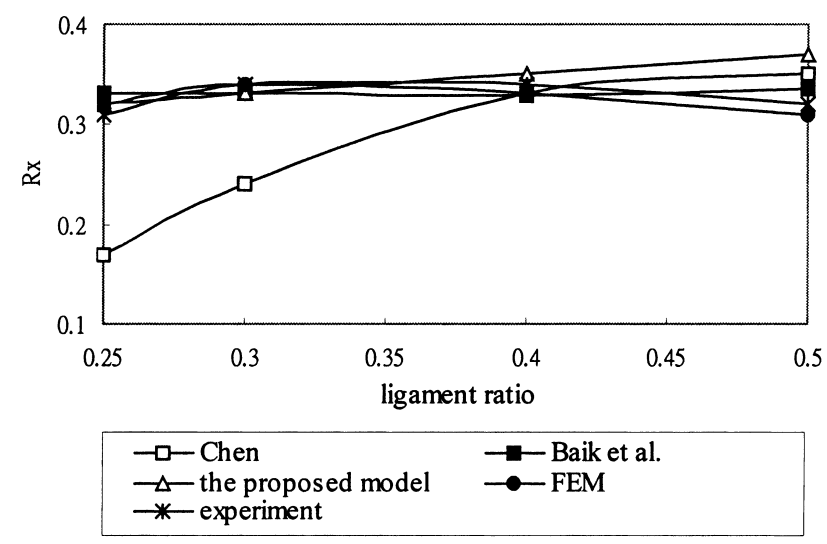

Fig. 11. Comparison of the apparent strain ratio $R_{x}$

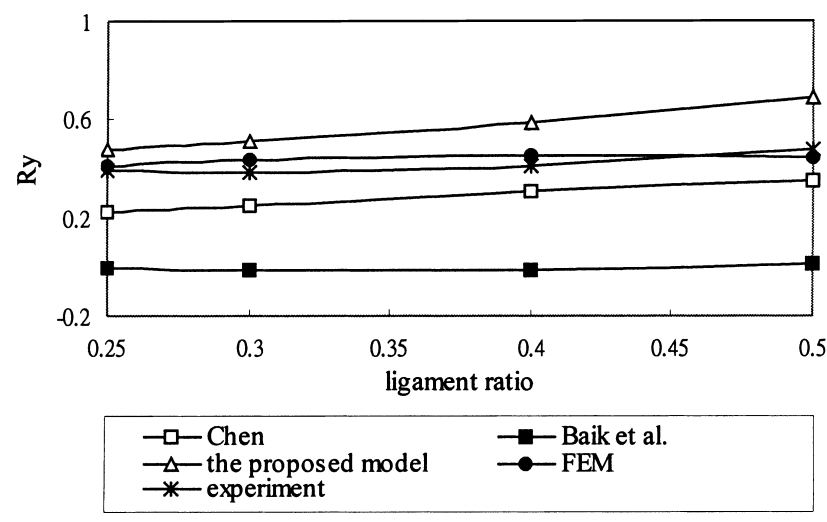

Fig. 12. Comparison of the apparent strain ratio $R_{y}$

ligament ratio. Hence, the apparent strain ratio $R_{x}$, under uniaxial loading in the $x$ direction, as predicted by Chen, is inappropriate for a perforated sheet with a lower ligament ratio, as shown in Fig. 11.

According to Fig. 12, the apparent strain ratio $R_{y}$ predicted by Baik et al. is almost zero and differs markedly from those obtained by Chen (this study) in the finite-element analysis and the experimental tests. This phenomenon is due to the correct factor of stress, $f$, used in Eq. (6), being close to zero, resulting in the very small coefficient of term $S_{x} S_{y}$ in Eq. (7). Hence, the apparent strain ratio $R_{y}$ derived by the yield criterion equation (7) is very small. On the other hand, under uniaxial loading in the $y$ direction, with the deformation model proposed by Chen, the yielding in the minimum ligament along $\mathrm{AB}$, is properly applicable for a perforated sheet with a larger ligament ratio, as shown in Fig. 9(b). Hence, the correlation of the apparent strain ratio $R_{x}$ predicted by Chen with those obtained by the finite-element analysis and the experimental tests is better than the results calculated by this study for a perforated sheet with a larger ligament ratio. However, when the ligament ratio of the perforated sheet is lower, the correlation of the apparent strain ratio $R_{x}$ predicted by this study with those obtained by the finite-element analysis and the experimental tests is better than the analytical results of Chen.

\section{Summary and concluding remarks}

The plastic behavior of a perforated sheet depends largely on the hole size and arrangement. Hence, the actual yielding condition of a perforated sheet is extremely complex and the yielding location is not fixed for various ligament ratios and different stress states. Hence, the assumptions of the yield location of a perforated sheet are not always practical over the whole range of ligament ratio.

In this study, perforated sheets with a uniform triangular pattern of round holes and low ligament ratios have been investigated. Yielding of the perforated sheets occurs close to the minimum ligament. Then, the stress components in 
the base metal across the minimum ligament width are calculated by the stress functions, their average values describing the yielding stress states of a perforated sheet. The von Mises yield criterion is assumed to govern the base metal of a perforated sheet. Next, the average values of stress components are substituted into the von Mises yield criterion to derive the yield criterion for a perforated sheet. Furthermore, the yield locus can be plotted by the yield criterion proposed herein. The yield locus is smooth and no singular point is present on the yield surface.

Simultaneously, the finite-element method and experiments were performed to discuss the validity of the yield criterion and the flow rules. The apparent yield stress ratios and the apparent strain ratios predicted by the proposed yield criterion are compared with those obtained by the finiteelement analysis, experiments and those predicated by Chen [4] and Baik et al. [7]. The apparent yield stress ratio, $Y_{x} / Y_{y}$, and the apparent strain ratios, $R_{x}$ and $R_{y}$, predicted by Chen are found to be inappropriate for a perforated sheet with a lower ligament ratio. This is because the assumption of a uniform stress distribution across the minimum ligaments is inapplicable. Although Baik et al. modified the analysis of the stress distributions across the minimum ligaments, the modified result is inappropriate for a low range of ligament ratio under uniaxial loading in the $y$ direction. Hence, the apparent strain ratio $R_{y}$ predicted by Baik et al. does not correlate well with those obtained by the finite-element analysis and the experiments. The present results indicate that the correlation of the apparent yield stress ratios and the apparent strain ratios predicted by this study with those obtained by the finite-element analysis and the experimental tests is better than the analytical results of Chen and Baik et al. In addition to those results correlating well with each other, the theoretical analysis in this study proposes a yield criterion in terms of apparent stresses and apparent strains, capable of accurately describing the plastic behavior of perforated sheets with a uniform triangular pattern of round holes and low ligament ratios.

\section{Acknowledgements}

The authors would like to thank the Industrial Technology Research Institute of the Republic of China for supporting this research. They also wish to thank Mr. Chih-Hung Chen for his assistance in performing the finite-element simulations and the experiments.

\section{References}

[1] K.A. Garnder, Heat exchanger tube sheet design, J. Appl. Mech., ASME 70 (1948) 377-385.

[2] E.E. Doerschuk, J.J. Moscony, D.M. Weber, Shadow-mask etching for data-display tubes, RCA Eng. 29 (2) (1984) 67-73.

[3] F.K. Chen, C. Hung, S. Kobayashi, Characterization of plastic deformation of sheet metals with square deformations, in: Proceedings of the 16th NAMRC, SME, North American Manufacturing Research, Institute of SME 1988, pp. 15-21.

[4] F.K. Chen, Analysis of plastic deformation for sheet metals with circular perforations, J. Mater. Process. Technol. 37 (1993) 175-188.

[5] S.C. Baik, K.H. Oh, D.N. Lee, Forming limit diagram of perforated sheet, Scripta Metall. Mater. 33 (1995) 1201-1207.

[6] S.C. Baik, K.H. Oh, D.N. Lee, Analysis of the deformation of a perforated sheet under uniaxial tension, J. Mater. Process. Technol. 58 (1996) 139-144.

[7] S.C. Baik, K.H. Oh, D.N. Lee, Plastic behaviour of perforated sheets under biaxial stress state, Int. J. Mech. Sci. 39 (1997) 781-793.

[8] W.J. O'Donnell, B.F. Langer, Design of perforated plates, J. Eng. Ind., Trans. ASME B 84 (1962) 307-320.

[9] G. Horvey, The plane-stress problem of perforated plates, J. Appl. Mech., Trans. ASME 74 (1952) 355-360.

[10] R. Hill, Proc. R. Soc. 193A (1948) 281.

[11] S.P. Timoshenko, J.N. Goodier, Theory of Elasticity, 3rd Edition, McGraw-Hill, New York, 1970, p. 57.

[12] M. Isida, Effect of width and length on stress intensity factors of internally cracked plates under various boundary conditions, Int. J. Fracture Mech. 7301.

[13] M. Isida, H. Igawa, Analysis of a zig-zag array of circular holes in an infinite solid under uniaxial tension, Int. J. Solids Struct. 27 (1991) 849-864.

[14] S.P. Tinoshenko, J.N. Goodier, Theory of Elasticity, 2nd ed. McGraw-Hill. 